Publicacions Matemátiques, Vol 36 (1992), 663-671.

\title{
ULTRAPRODUITS GRADUÉS. APPLICATIONS
}

\author{
ION D. ION ET CONSTANTin Nitä*
}

Dédié à la mémoire de Pere Menal

\begin{abstract}
Dans cet travail sont défnis les uitraproduits d'anneaux et de modules gradués. L'ultraproduit gradué coincide dans le cas d'une famille $R_{2}\left[X_{1}, X_{2}, \ldots, X_{n}\right], i \in I$, d'anneaux de polynômes avec le sousanneau des éléments générés dans l'ultraproduit usuel par les familles de polynômes de degré total borné.

Nous démonstrons que l'ultraproduit d'une famille de modules gradués libres, qui verifie une condition naturelle de finitude et aussi un module gradué libre (Théorème 2.2). Après un etude de l'arithmétique des monoides commutatifs par rapport aux ultraproduits, nous démontrons que tout ultraproduit d'une famille d'extensions de Galois de degré borné est une extension de Galois de degré fini (Théorème 3.3).
\end{abstract}

\section{Introduction}

Les logiciens ont introduit et ont utilisé ultérieurement la notion d'ultraproduit dans leurs travaux. Amitsur a montré pour la première fois que cette notion peut-être utilisé dans la théorie des anneaux. Un application de cette idée est, par exemple, la démonstration d'un théorème de Posner qui décrit la structure des anneaux premiers qui satisfaient une identité polynomiale. Il est bicn-connu que la théorie des ultraproduits a dévêloppé une technique trc̀s efficace dans l'algèbre commutative, tout particulièrement, pour les théorèmes d'approximation. Dans l'algèbre non-commutative les applications des ultraproduits sont plus ou moins accidentelles. Notre intention est d'élargir le domaine d'applicabilité de cette technique dans les problèmes d'algèbre non-commutative, particulièrement dans la théorie des anneaux gradués. Nous avons essayé une contribution dans cette direction. Dans la suite, sont donnés quelques résultats des auteurs, qui son réunis entre autres dans les travaux [4] et [5]. Au commencement, nous désirons rappeler un merveilleux travail [3], très utile dans ce domain des ultraproduits en génèral.

*Conférence presentèe par M.C. Niţă a "International Workshop on Local Cohomology, Geometric Applications and Related Topics", Granada, Sept. 1991. 


\section{Géneralités sur les ultraproduits d'anneaux et modules}

D'abord en deux mots on donnent quelques définitions sur l'ultraproduit usuel.

Soient $I$ un ensemble infini et $F$ un ultrafiltre non-principal sur $I . \mathrm{Si}$ $\left(R_{i}\right)_{i \in I}$ est une famille d'anneaux unitaires, on note $R=\prod_{i \in I} R_{i}$. Pour $a \in R, a=\left(a_{i}\right)$ soit $Z(a)=\left\{i \in I \mid a_{i}=0\right\}$. Alors l'ensemble $Z_{F}(R)=$ $\{a \in R \mid Z(a) \in F\}$ est un idéal idempotent de l'anneau $R$. L'anneau quotient $R_{F}=R / Z_{F}(R)$ s'appele l'uitraproduit de la famille $\left(R_{i}\right)_{i \in I}$ par rapport a $F$. Notons $a^{*}$ l'image de $a \in R$ par le morphisme canonique $R \rightarrow R_{F}$.

D'une manière analogue, on définit l'ultraproduit d'une familie de modules $\left(M_{i}\right)_{i \in I}, M_{i} \in R_{i}$-mod. Soit $M=\prod_{i \in I} M_{2} \in R$-mod. L'ensemble $Z_{F}(M)=\{x \in M \mid Z(x) \in F\}$ est un sous-module de $M$ et le module quoticnt s'appele l'ultraproduit de la famille $\left(M_{i}\right)_{i \in I}$ par rapport à $F$. Parce que $Z_{F}(R) M \subset Z_{F}(M)$, alors $M_{F}$ est muni d'une structure de module sur $R_{F}$.

Proposition 1.1. Soit $\mathcal{C}_{F}=\left\{N \in R-\bmod \mid Z_{F}(R) \subset \operatorname{ann}_{R} N\right\}$. Alors $\mathcal{C}_{F}$ est un sous-catégorie localisante de $R$-mod, isomorphe à la catégorie $R_{F}-$ mod.

Soient $M=\prod_{i \in I} M_{i}, N=\prod_{i \in I} N_{i}$, ou $M_{i}, N_{i} \in R_{i}$ mod pour tout $i \in I$. Si $f \in \operatorname{Hom}_{R}(M, N), f=\left(f_{i}\right)$, où $f_{i} \in \operatorname{Hom}_{R_{i}}\left(M_{i}, N_{i}\right), i \in I$, alors l'application $f_{F}: M_{F} \rightarrow N_{F}, f_{F}\left(x^{*}\right)=\left(f_{i}(x)\right)^{*}, x \in M, x=\left(x_{i}\right)$ est l'unique morphisme dans $\operatorname{Hom}_{R_{F}}\left(M_{F}, N_{F}\right)$ tel que $\varphi_{N} \circ f=f_{F} \circ \varphi_{M}$, où $\varphi_{M}: M \rightarrow M_{F}, \varphi_{N}: N \rightarrow N_{F}$ sont les morphismes canoniques.

La correspondance $M \rightarrow M_{F}$ a un caractère foncteuriel.

Proposition 1.2. Si pour tout $i \in I$,

$$
0 \longrightarrow M_{i}^{\prime} \longrightarrow M_{i} \longrightarrow M_{i}^{\prime \prime} \longrightarrow 0
$$

est une suite exacte (exacte est scindée) dans $R_{i}$-mod, alors

$$
0 \longrightarrow M_{F}^{\prime} \longrightarrow M_{F} \longrightarrow M_{F}^{\prime \prime} \longrightarrow 0
$$

est un suite exacte (exacte et scindée) dans $R_{F}$-mod. 


\section{Ultraproduits gradués}

$\mathrm{Si}$ on considère $\left(R_{i}\left[X_{1}, X_{2}, \ldots, X_{n}\right]\right)_{i \in I}$ une famille d'anneaux de polynômes, il existe un morphisme canonique

$$
\varphi: R_{F}\left[X_{1}, X_{2}, \ldots, X_{n}\right] \longrightarrow\left(\prod_{i \in I} R_{i}\left[X_{1}, X_{2}, \ldots, X_{n}\right]\right)_{F}
$$

qui n'est pas un isomorphisme. On a donc qu'un ultraproduit d'une famille d'anneaux de polynômes n'est pas un anneau de polynômes (voir, par exemple [3], dans une démonstration d'un résultat de Robinson). L'image de $\varphi$ est un sous-anneau de l'ultraproduit $\left(\prod_{i \in I} R_{i}\left[X_{1}, X_{2}, \ldots, X_{n}\right]\right)_{F}$. Les éléments de cet sous-anneau sont representés par les familles bornées de polynômes.

Ici on trouve l'idée de construire un ultraproduit gradué. Les ultraproduits gradués d'algèbre de polynômes donnent un cadre naturel pour une série d'applications.

Dans la suite on présente la construction d'ultraproduit gradué. Soit $G$ un monoïde tel que tout élément $\sigma$ de $G$ est régulier. Pour tout $i \in I$, soit $R_{i}$ un anneau gradué de type $G$ :

$$
R_{i}=\bigoplus_{\sigma \in G} R_{i \sigma}, \quad R_{i \sigma} R_{i \tau} \subset R_{i \sigma \tau}, \quad \forall \sigma, \tau \in G
$$

Soient $R=\prod_{i \in I} R_{i}$ et $R^{g}=\bigoplus_{\sigma \in G}\left(\prod_{i \in I} R_{i \sigma}\right) \subset R$. Notons $R_{\sigma}^{g}=\prod_{i \in I} R_{i \sigma}, \sigma \in$ $G$. Alors $R_{\sigma}^{g} R_{\tau}^{g} \subset R_{\sigma \tau}^{g}$ et donc $R^{g}$ est un anneau gradué de type $G$. Il est facile de voir que $Z_{F}^{g}(R)=R^{g} \cap Z_{F}(R)$ est un idéal gradué de $R^{g}$. L'anneau gradué $R_{F}^{g}=R^{g} / Z_{F}^{g}(R)$ est appelé l'ultraproduit gradué de la famille $\left(R_{i}\right)_{i \in I}$. On a une définition analogue pour l'ultraproduits gradués d'une familie $\left(M_{i}\right)_{i \in I}$ de modules gradués de type $G$ :

$$
M_{i}=\bigoplus_{\sigma \in G} M_{i \sigma}, \quad R_{i \sigma} M_{i \tau} \subset M_{i \sigma \tau}, \quad \forall \sigma, \tau \in G
$$

Soient $M=\prod_{i \in I} M_{i}, M^{g}=\bigoplus_{\sigma \in G}\left(\prod_{i \in I} M_{i \sigma}\right) \subset M, Z_{F}^{g}(M)=Z_{F}(M) \cap M^{g}$.

On a que $M^{g}$ est un $R^{g}$-module gradué de type $G$ et $Z_{F}^{g}(M)$ est un $R^{g}$-sousmodule gradué de $M^{g}$. Alors $M_{F}^{g}=M^{g} / Z_{F}^{g}(M)$ s'appele I'ultraproduit gradué de la famille $\left(M_{i}\right)_{i \in I}$ de modules gradués. Parce que $Z_{F}^{g}(R) M^{g} \subset Z_{F}^{g}(M)$, alors $M_{F}^{g}$ est muni d'une structure de $R_{F}^{g}$ module. 
On dit qu'un élément $a \in R=\prod_{i \in I} R_{i}, a=\left(a_{i}\right)$ est de $G$-support fini, s'il existent $U \in F$ et $\sigma_{1}, \sigma_{2}, \ldots, \sigma_{n} \in G$, tels que $a_{i} \in R_{i \sigma_{1}}+R_{i \sigma_{2}}+\cdots+R_{i \sigma_{n}}$, pour tout $i \in U$.

Dans la démonstration des certains résultats donnés dans la suite est importante la suivante:

Proposition 2.1. L'ensemble $\left\{a^{*} \mid a \in R\right.$, a avec le G-support fini $\}$ est un sous anneau de $R_{F}$, isomorphe $\dot{a} R_{F}^{g}$.

Soit $S$ un ensemble d'éléments homogènes non-nuls d'un module gradué. Pour tout $\sigma \in G$ soit $S_{\sigma}=\{x \in S \mid \operatorname{deg} x=\sigma\}$. On dit que deux ensembles $S$ et $S^{\prime}$ d'éléments homogènes nonuls sont $g r$-similaires si $S_{\sigma}$ et $S_{\sigma}^{\prime}$ ont le même cardinal pour tout $\sigma \in G$. Dans ce cas on peut ecrire $S=\left(x_{t}\right)_{t \in T^{\prime}}, S^{\prime}=\left(x_{t}^{\prime}\right)_{t \in T}$ tel que $\operatorname{deg} x_{t}=\operatorname{deg} x_{t}^{\prime}$ pour tout $t \in T$.

Dans ce cas on dit que les ensembles $S$ et $S^{\prime}$ sont $g r$-indexés d'un façon compatible.

Un resultat principal est le suivant:

Théorème 2.2. Soit $\left(R_{i}\right)_{i \in I}$ une famille d'anneaux gradués de type G. Pour tout $i \in I$, soit $L_{i}=\bigoplus_{\sigma \in G} L_{i \sigma}$ un $R_{i}$-module gradué ayant un base homogène $B_{i}$. Supposons que les bases $B_{i}, i \in I$, sont gr-similaires, $B_{i}=\left(e_{i t}\right)_{t \in T}, \operatorname{deg} e_{i t}=\operatorname{deg} e_{j t}$, pour tous $i, j \in I$ et $t \in T$ (c'est-à-dire, les bases sont $g r$-indexées d'un façon compatible). Si pour tout $\sigma \in G$, il existe $d \in \mathbb{N}^{*}$ et $t_{1}, t_{2}, \ldots, t_{d} \in T$ tels que on a la condition de finitude:

$$
L_{i \sigma} \subset R_{i} e_{i t_{3}}+R_{i} e_{i t_{2}}+\cdots+R_{i} e_{i t_{d}}, \quad i \in I,
$$

alors pour tout ultrafiltre nonprincipal $F$ sur $I, L_{F}^{g}$ est un $R_{F}^{g}$-module gradué libre et $B_{F}=\left(e_{t}^{*}\right)_{t \in T}$, où $e_{t}=\left(e_{i t}\right)$ est une $R_{F}^{g}$-base sur $L_{F}^{g}, g r$ similaire avec chaque base $B_{i}, i \in I$.

Dans le cas de monoïde $(\mathbb{N},+)$ et de la graduation triviale sur $R_{i}$ et $L_{i}$ on obtient

Corollaire 2.3. Soit $\left(R_{i}\right)_{i \in I}$ une famille infinie d'anneaux unitaires. Pour tout $i \in I$ soit $L_{i}$ un $R_{i}$-module ayant un base à n éléments. Alors:

1) $[8] L_{F}$ est un $R_{F}$-module libre ayant une base à $n$ éléments.

2) $\operatorname{End}_{R_{F}}\left(L_{F}\right)$ est isomorphe à $\left(\prod_{i \in I} \operatorname{End}_{R_{i}}\left(L_{i}\right)\right)_{F}$.

Corollaire 2.4. [9] Soit $\left(R_{i}\right)_{i \in I}$ une famille d'anneaux unitaires. Pour tout $n \in \mathbb{N}$ on a que $M_{n}\left(R_{F}\right)$ est isomorphe $a ̀\left(\prod_{i \in I} M_{n}\left(R_{i}\right)\right)_{F}$. 
It est bien-connu [6] qu'un ultraproduit de corps est, de même, un corps. Pour le cas des anneaux simples un tel résultat n'est pas vraie [1]. D'après le corollaire précédent, il resulte:

Si $\left(S_{i}\right)_{i \in I}$ est une famille d'aneaux simples tel que $S_{i}$ est isomorphe a $M_{n}\left(K_{i}\right), i \in I$ (pour le même ordre $n$ ) où $K_{i}$ est, un corps pour tout $i \in I$, alors chaque ultraproduit de $\left(S_{i}\right)_{i \in I}$ est encore un anneau simple isomorphe à $M_{n}\left(\left(\prod_{i \in I} K_{i}\right)_{F}\right)$.

Corollaire 2.5. Pour tout $i \in I$ soit $P_{i}$ un $R_{i}$-module projectif oyant un système de générateurs à $n$ elements. Alors $P_{F}$ est un $R_{F}$-module projectif de type fini.

Pour les algèbres de polynômes on utilise la graduation donnée par le degré total. On a:

Corollaire 2.6. Pour tout $i \in I$ soit $A_{i}$ une $R_{i}$-algèbre commutative. $S i\left(x_{i 1}, x_{i 2}, \ldots, x_{i d}\right)$ est un système d'éléments de $A_{i}, i \in I$, algèbriquement indépendants sur $R_{i}$, alors les éléments $x_{k}^{*}=\left(x_{i k}\right)^{*} \in$ $R_{F}, 1 \leq k \leq d$, son algèbriquement indépendants sur $R_{F}$. De plus, l'ultraproduit gradué des algèbres $R_{i}\left[x_{i 1}, x_{i 2}, \ldots, x_{i d}\right], i \in I$, est isomorphe à l'algèbre $R_{F}\left[x_{1}^{*}, x_{2}^{*}, \ldots, x_{d}^{*}\right]$.

Donc, considerant la graduation donnée par le degré total pour l'algèbre de polynômes, on a que $R_{F}\left[X_{1}, X_{2}, \ldots, X_{d}\right]$ est isomorphe $\grave{a}$ $\left(\prod_{i \in I} R_{i}\left[X_{1}, X_{2}, \ldots, X_{d}\right]\right)_{F}^{g}$, où $\left(R_{i}\right)_{i \in I}$ est une famille d'anneaux commutotifs et $F$ un ultrafiltre nonprincipal sur $I$.

\section{Divisibilité et ultraproduits}

Quelques applications des ultraproduits imposent l'étude de la factorialité par rapport aux ultraproduits. La factorialité peut-être aborder soit pour monoïdes soit pour anneaux d'integrité. Dans l'étude de la factorialité pour monoïdes il est intéréssant de voir comme intervient d'un façon naturel, l'idée d'ultraproduit gradué.

Il est connu qu'un ultraproduit usuel d'anneaux factoriels n'est pas toujours un anneau factoriel [10].

Quoique on peut utiliser la construction deja faite dans la section 1 pour obtenir l'ultraproduit d'une famille d'anneaux d'integrité, nous rappelons en deux mots la construction d'ultraproduit pour monoides avec simplification. 
Soit $\left(M_{i}\right)_{i \in J}$ une famille de monoides commutatifs dont tous les éléments sont réguliers. Si $a, b \in \prod_{i \in I} M_{i}, a=\left(a_{i}\right), b=\left(b_{i}\right)$ on pose:

$a \sim_{F} b$ si et seulement si $\left\{i \mid a_{i}=b_{i}\right\} \in F$.

Alors la rélation $\sim_{F}$ est une rélation d'équivalence sur le monoïde $M$, compatible avec le produtit. Le monö̈de quotient $M_{F}=M / \sim_{F}$ est appelé l'ultraproduit de la famille $\left(M_{i}\right)_{i \in I}$ de monoïdes. On voit que tous les éléments du monoïde $M_{F}$ son réguliers. On note par $U(M)$, le groupe des éléments inversables du monoïde $M$.

Lemme 3.1. Avec les notations précédentes, on a:

1) $U\left(M_{F}\right)=\left(\prod_{i \in I} U\left(M_{i}\right)\right)_{F}$;

2) L'élément $p^{*} \in M_{F}, p=\left(p_{i}\right) \in \prod_{i \in I} M_{i}$, est un élément irreductible (premier) dans $M_{F}$ si et seulement si $\left\{i \mid p_{i}\right.$ irreductible (premier) dans $\left.M_{i}\right\} \in F$.

Les choses précédentes peut-être données pour anneaux d'integrité dont ultraproduit a été defini ci-dessus.

Soient maintenant les monoildes $(M, \cdot)$ et $(G, \cdot)$. On dit que $M$ est un monoïde $G$-gradué s'il existe une famille de sous-esembles $\left\{M_{\sigma}\right\}_{\sigma \in G}$ de $M$ tel que $M=\bigcup_{\sigma \in G} M_{\sigma}, M_{\sigma} M_{\tau} \subset M_{\sigma \tau}$ pour tout $\sigma, \tau \in G$ et $M_{\sigma} \cap M_{\tau}=$ $\phi$ pour tout $\sigma \neq \tau$.

Exemple. Soit $M$ un monoïde factoriel, $(U, \cdot)$ le groupe des éléments inversables de $M$. Pour tout $n \in \mathbb{N}$ on défini $M_{n}=\{x \in M \mid l(x)=n\}$, où $l(x)$ est la longeur d'élément $x$. On a que $M$ est un monoïde $\mathbb{N}$-gradué et $M_{0}=U(M)$.

Un sousmonoïde $(N$,$) de monoïde gradué (M, \cdot), M=\bigcup_{\sigma \in G} M_{\sigma}$ est appelé $g r$-sousmonoìde de $M$ si $N=\bigcup_{\sigma \in G}\left(M_{\sigma} \cap N\right)$.

Soient $\left(M_{i}\right)_{I \in I}$ une famille infinie de monödes $G$-gradućs, $M_{i}=\bigcup_{\sigma \in G} M_{i \sigma}$ et $F$ un ultrafiltre nonprincipal sur $I$. Si $M^{g}=\bigcup_{\sigma \in G} M_{\sigma}$, où $M_{\sigma}=$ $\prod_{i \in I} M_{i \sigma}, \sigma \in G$, alors $M^{g}$ est un sousmonoïde de $M$; il est clair que $\left(M^{g}, \cdot\right)$ est muni d'une structure de monö̈de $G$-gradué. Evidement, $M_{F}^{g}=M^{g} / \sim_{F}=\left\{a^{*} \in M_{F} \mid a \in M^{g}\right\}$ est un sousinonoïde de $\left(M_{F},{ }^{\prime}\right)$ et $\left(M_{F}^{g}, \cdot\right)$ est muni d'une structure de monoïde $G$-gradué. Le monö̈de $\left(M_{F}^{g}, \cdot\right)$ s'appele le $g r$-ultraproduit de la famille $\left(M_{i}\right)_{i \in I}$ de monoïdes gradues. 
Soit $(M, \cdot)$ un monoïde commutative et $(S, \cdot)$ un sousmonoïde de $M$. Une famille $\left(x_{t}\right)_{t \in T}$ contenue dans $M \backslash S$, s'appele un système libre de générateurs (sur $S$ ) si tout element $x \in M$ s'ecrit d'un façon unique $x=a x_{t_{1}}^{e_{1}} x_{t_{2}}^{e_{2}} \ldots x_{t_{d}}^{e_{d}}, d \geq 0, e_{i} \in \mathbb{N}^{*}, a \in S$. Alors $M$ est un monoïde $\mathbb{N}$-gradué, où la graduation cst donnée par:

(*) $M_{n}=\left\{a x_{t_{1}}^{e_{1}} x_{t_{2}}^{e_{2}} \ldots x_{t_{d}}^{e_{d}} \mid a \in S, e_{2} \in \mathbb{N}^{*}, \sum_{i=1}^{d} e_{i}=n\right\}, \quad n \in \mathbb{N}$.

Exemple. $\mathrm{Si}(M, \cdot)$ est un monoïde factoriel, $U=U(M)$ est le groupe d'éléments inversables, $\left(p_{i}\right)_{i \in T}$ est un système representatif d'éléments premiers, alors $\left(p_{i}\right)_{i \in T}$ est un système libre de générateurs (sur $U$ ) du monoïde $M$.

Il existe un théorème pour les monoïdes gradués analogue à celui énoncé pour les modules libres.

Théorème 3.2. Soit $\left(M_{i}\right)_{i \in I}$ une famille infinie de monö̈des commutatifs tel que chaque monoüde $M_{i}$ admet un système libre de générateurs $\left(x_{i_{1}}, x_{i_{2}}, \ldots, x_{i_{d}}\right)$ sur le sousmonoüde $S_{i}$ de $M_{i}, d \in \mathbb{N}^{*}$. Si chaque $M_{i}$ est gradué avec la graduation (*), alors pour tout ultrafilter $F$ sur $I, M_{F}^{g}$ admet un système libre de générateurs sur sousmonoïde $S_{F}$.

Ce théorème montre que dans certains cas de graduation pour les monoïdes factoriels, la proprićté de factorialité se conserve par des ultraproduits.

Soient $\left(M_{i}\right)_{i \in J}$ une famille infinie de monoïdes factoriels, $P_{i}$ un système representatif d'éléments premiers. On considère que $M_{i}, i \in I$, est $\mathbb{N}$ gradué par rapport à la longeur des éléments.

Si $F$ est un ultrafiltre nonprincipal sur $I$, alors pour tout $a^{*} \in M_{F}$, avec $a$ de longuer bornée, il existe $d$ tel que $\left\{i \mid l\left(a_{i}\right) \leq d\right\} \in F$.

Soit $P^{*}$ l'ensemble des éléments premiers du monoìde $M_{F}, U^{*}$ l'ensemble des éléments inversables. On a, clairement, que $\operatorname{Fact}\left(M_{F}\right) \stackrel{\text { def }}{=}$ $\left\langle U^{*}, P^{*}\right\rangle$ (c'est-à-dire le sousmonoïde de $M_{F}$ engendrée par $U^{*}$ et $P^{*}$ ) est un monoïde factoriel.

On considère que chaque $M_{i}$ est $\mathbb{N}$-gradué:

$$
M_{2}=\bigcup_{n \geq 0} M_{i n}, \quad M_{i n}=\left\{x_{i} \in M_{i} \mid l\left(x_{i}\right)=n\right\} .
$$

Théorème 3.3. Dans les conditions précédentes on $a$ :

1) $\operatorname{Fact}\left(M_{F}\right)=\left\{a^{*} \in M_{F} \mid a=\left(a_{i}\right)\right.$ a longuer bornée $\}$.

2) $\operatorname{Fact}\left(M_{F}\right)$ est isomorphe $a\left(\prod_{i \in I} M_{i}\right)_{F}^{g}$. 


\section{Ultraproduits d'extensions de Galois}

L'étude des uitraproduits d'extensions de Galois a imposé l'étude de la factorialité par rapport aux ultraproduits.

Soient $\left(L_{i} / K_{i}\right)_{i \in I}$ une famille d'extensions de corps commutatifs. Si pour tout $i \in I$ on a $\left[L_{i}: K_{i}\right]=n$, alors d'après le corollaire 2.3 il résulte $\left[L_{F}: K_{F}\right]=n, F$ étant un ultrafiltre non-principal sur $I$.

Ci-dessus, ont été étudić entre autres la rélation de divisibilité dans les anneaux $K_{F}[X]$ et $L_{F}[X]$ et les polynomes irreductibles dans ces anneaux. On pose de même le problème de multiplicité des racines pour polynômes. Uitilisant les résultats d'arithmetique pour monoïdes factoriels, on peut caractériser le polynôme minimal sur $K_{F}$ d'une élément $\alpha^{*} \in L_{F}, \alpha=\left(\alpha_{i}\right), \alpha_{i} \in L_{i}, i \in I$, avec les polynômcs minimaux de $\alpha_{i}, i \in I$, sur $K_{i}$. On a

Lemme 4.1. Si $L_{i} / K_{i}$ est une extension separable de degré n pour tout. $i \in I$, alors $L_{F} / K_{F}$ est une extension separable de degrén.

Lemme 4.2. Si $L_{i} / K_{i}$ est une extension normale de degré $n$, pour tout $i \in I$, alors $L_{F} / K_{F}$ est une extension normale de degré $n$.

Le. résultat principal de cette section est le suivant:

Théorème 4.3. Soit $\left(L_{i} / K_{i}\right)_{i \in I}$ une famille d'extensions de Galois de degré n. Alors:

1) $L_{F} / K_{F}$ est une extension de Galois de degré $n$.

2) $\operatorname{Gal}\left(L_{F} / K_{F}\right)$ est isomorphe $\dot{a}\left(\prod_{i \in I} \operatorname{Gal}\left(L_{i} / K_{i}\right)\right)_{F}$.

\section{References}

1. U. Bartocci, Sulla generalizzazione di un teorema di Kochen, Rend. Accad. Naz. Linci XIV (1968), 221-230.

2. J. Becher, J. Denef, L. LiPSCHITZ AND L. DRIES, Ultraproducts and approximation in local rings, I. Inventiones Math. 51. (1979), $189-205$.

3. P. EKLOF, "Ultraproducts for algebraists. Handbook of Mothematical Logic," ed. by J. Barwise, North-Holland, 1977.

4. I. D. ION AND C. Niq̧, Graded ultraproducts, Rev. Roum. Math. Pures Appl. 35, 1 (1990), 4148. 
5. I. D. ION AND C. Niţă, Ultraproducts of field extensions, Bull. Math. de la Sac. Math. de Roumanie tome 34 (82), nr. 3 (1990), 247-254.

6. S. KoChen, Utraproducts in the theory of models, Ann. of Math., Ser. 2, 74 (1961), 221-261.

7. C. NĂSTĂSESCU AND F. VAN OYSTAEYEN, "Graded ring theory," North-Holland, 1982.

8. A. Nị̧ă, Ultraproducts of some classes of modules, Bull. I. P. Bucuresti, seria Automatică (1988).

9. A. Nǐ̧̧̆ ET C. Niţă, Sur l'ultraproduit des anneaux de matrices, Bull. Math. de Soc. Sci. Math. de Roumanie tome 29 (77), nr. 3 (1985), 275-278.

10. G. SAnTosuosso, Sul transporto ad un ultraprodotto di anelli di proprieta dei suoi fattori, Rend. Math. 6, I (1968), 82-100.

\author{
Faculté de Mathématiques \\ Université de Bucarest \\ Str. Academiei, nr. 14 \\ Bucarest \\ ROMANIA
}

Rebut el 3 d'Abril de 1992 